\title{
Uso de tecnologías en las infancias: pautas transaccionales y estrategias de socialización familiares
}

\section{Use of technologies in children: transactional guidelines and socialization strategies in families}

\author{
Recibido el: 01/03/2021 \\ Aprobado el: 04/06/2021
}

\author{
Paula Gabriela Irueste ${ }^{1}$ \\ María Barotto ${ }^{2}$ \\ Sofía Samanón ${ }^{3}$ \\ Melisa Belén Taormina ${ }^{4}$
}

\begin{abstract}
Resumen
Este estudio se realizó en el año 2019 y buscó conocer el uso de tecnologías en niños y niñas de entre 4 y 12 años de edad, respecto a las pautas transaccionales y las estrategias de socialización implementadas por las familias. Se realizó un estudio de tipo mixto, utilizando una entrevista semiestructurada y un cuestionario online construido "ad hoc". La población estudiada fueron personas a cargo (padres, madres, tutores) de niños y niñas de entre 4 y 12 años. Los resultados revelaron que los/as infantes hacían uso de aparatos tecnológicos entre dos y tres horas diarias, desde los primeros meses o años de vida. Los adultos también los usaban y solía ser en presencia de los/as infantes. El diálogo y los acuerdos eran escogidos como estrategias para comunicar los límites, y los sostienen en el tiempo. Por último, los adultos manifestaron como apropiado el uso de las pantallas por parte de los/as infantes y la estrategia que predominaba, para regular el uso, es la de pautar horarios.
\end{abstract}

Palabras claves: uso de tecnologías, familias, pautas transaccionales, estrategias de socialización, infancias

\begin{abstract}
The present study has been made in 2019 and focuses on learning about the use of technology in children between 4 and 12 years of age, concerning transactional guidelines and socialization strategies implemented by families. We carried out a mixed type of study, and we used two types of tools: a semistructured interview and an anonymous Ad hoc questionnaire. The studied population corresponds to persons in charge (fathers, mothers, tutors) of the children between 4 and 12 years. The achieved results revealed that infants made use of technological devices for between two and three hours every day from the early months or years of their life. Adults also made use of these devices and it often was

\footnotetext{
${ }^{1}$ Licenciada en Psicología, Directora del Servicio de Neuropsicología Área Infantil, Facultad de Psicología, UNC. Córdoba, Argentina. paula.irueste@unc.edu.ar. Porcentaje de autoría:25\% ${ }^{2}$ Licenciada en Psicología, Profesional Invitada del Servicio de Neuropsicología Área Infantil, Facultad de Psicología, UNC. Córdoba, Argentina. mariabarotto4@gmail.com. Porcentaje de autoría: $25 \%$

${ }^{3}$ Licenciada en Psicología, Profesional Invitada del Servicio de Neuropsicología Área Infantil, Facultad de Psicología, UNC. Córdoba, Argentina. sofiasamanon@gmail.com. Porcentaje de autoría: $25 \%$

${ }^{4}$ Licenciada en Psicología, Profesional Invitada del Servicio de Neuropsicología Área Infantil,
}

Facultad de Psicología, UNC. Córdoba, Argentina.melitaor@gmail.com. Porcentaje de autoría:25\%
\end{abstract}


in front of the children. Dialogue and agreements were implemented to communicate the limits, and once shared, they are sustained over time. By last, adults revealed they found the children's use of the technologies to be appropriate, setting a schedule was the strategy that prevails to reduce the usage.

Keywords: use of technologies, families, transactional guidelines, socialization strategies, childhood

\section{Introducción}

En la actualidad, nos encontramos atravesados por las tecnologías, un fenómeno con el que estamos en permanente contacto e impregna todas las áreas y ámbitos de nuestra vida, siendo nosotros los seres humanos, quienes le otorgamos tal relevancia. Hablamos de una revolución tecnológica, definida por su capacidad de penetración en todos los ámbitos del ser humano y por el procesamiento de la información, el conocimiento y la comunicación. Pero, además, aparecen nuevas configuraciones tempo-espaciales, haciendo que se produzcan nuevos entornos y nuevos vínculos en relación a la virtualidad, que hasta entonces eran desconocidos. (Iriarte Díazgranados, 2011)

En esta misma línea, las familias también atraviesan transformaciones y por ende, sienten la necesidad de reconfigurarse para una mayor adaptación y funcionalidad a estos cambios.

Considerando que "las prácticas con las tecnologías no son neutrales, no tienen el mismo significado para todos, y no pueden ser analizadas de forma aislada sin tener en cuenta el entorno de cada niño, su uso se integra en el estilo de vida y en los esquemas familiares y son apropiadas de forma diferente" (Martínez-Piñeiro, Couñago, y Barujel, 2018, p. 2). En la presente investigación, realizada en el año 2019, se buscó conocer el uso de las tecnologías en las infancias, respecto a las pautas transaccionales y las estrategias de socialización, implementadas por las diferentes familias.

\section{Familias y tecnologías}

La familia, puede ser definida no sólo como un conjunto de personas en permanente interacción, regulada tanto por reglas internas como por funciones dinámicas que existen entre sí y con el mundo externo (Minuchín et al., en Espinal, Gimeno y González, 2006); sino además como un "sistema activo en transformación constante" (Andolfi, 1984, p.76). La caracterizamos como un sistema abierto, ya que sus límites son permeables a la influencia de otros sistemas. Además, al ser un conjunto, una totalidad, decimos que se construye y sostiene por un sistema de valores y creencias compartidas, por costumbres, rituales, modos de vincularse y experiencias vividas por sus miembros, ya sea a nivel individual o grupal; creando así, una identidad familiar y reforzando el sentido de pertenencia. Hablamos de que presenta una interacción circular y bidireccional, debido a que los intercambios conllevan una mutua influencia entre sus miembros (Espinal, Gimeno, y González, 2006).

Como mencionamos anteriormente, las relaciones familiares tanto internas como externas, son reguladas por reglas que funcionan de manera explícita o implícita. Estas, evidencian la funcionalidad del sistema debido a que son acordes a las creencias y valores familiares y por ende, condicionan las necesidades, conductas y la comunicación de sus miembros. Las normas familiares son elaboradas y mantenidas por las figuras que asumen el liderazgo del sistema, quienes también 
ejercen mayor influencia en los modos de vincularse, pensar, sentir y actuar de los diferentes miembros. Minuchín (1982), sostiene que mientras mayor democracia y flexibilidad haya en la manera de liderar, la funcionalidad del sistema también aumentará. Además, la familia es un sistema autoorganizado, porque plantea sus propias metas y medios para alcanzarlas, así como también, autogestiona su evolución mediante estrategias, normas, recursos y procedimientos implementados por sus miembros en las diferentes situaciones que atraviesan.

Buscando ampliar nuestra concepción del sistema familiar, consideramos que no podemos dejar de lado que éste ha ido mutando a lo largo de la historia, viéndose atravesado no sólo por las realidades individuales de sus miembros, sino también por el contexto socioeconómico y cultural (De Jong, Basso, Paira y García, 2009). Como ya sabemos, actualmente, dicho contexto está mediado por las tecnologías, las cuales intervienen en gran medida en la cotidianeidad familiar, generando modificaciones en las interacciones entre infantes y cuidadores, a las que cada sistema familiar puede o no tener la capacidad de adaptarse. Es decir, se incorporan como un nuevo integrante en las dinámicas familiares, y el uso que hacen los adultos o los/as infantes de las tecnologías influirá en las mismas.

Cuando hablamos de Uso de Tecnologías, lo definimos como todo contacto cotidiano con diferentes aparatos tecnológicos, de manera activa o pasiva, y con diferentes finalidades, tales como realizar actividades académicas, de entretenimiento y ocio o de comunicación interpersonal. Además, algo que caracteriza al uso de las tecnologías, es el concepto de interactividad, como aquella posibilidad de participar activamente en el proceso de comunicación de los participantes (Vodopivec y Samec, 2013). Los factores que nos permiten observar este uso son, la frecuencia con la que acceden a las tecnologías, la cantidad de horas dedicadas a su uso, la finalidad con la que lo hacen, la edad a la que comenzaron a usarlas y las restricciones, puestas o no, por los adultos respecto al acceso de los infantes a las mismas.

Con el auge de las tecnologías, el internet interviene en gran medida en la cotidianeidad familiar, generando modificaciones en las interacciones entre infantes y cuidadores. En lo que a los últimos respecta, definimos tres tipos de usos y habilidades respecto a internet; a saber, el que no hace uso directo de internet ni de la computadora, es decir, establecen una relación indirecta con los mismos mediante la regulación del uso que niños/as hacen de estos dispositivos, controlando la dinámica familia-internet. En segundo lugar, tenemos aquellos que sí hacen uso directo, pero con limitaciones, son quienes esporádicamente se conectan por sí solos a la red, ya que recurren a los/as niños/as, tanto para encender la computadora como para ingresar al mail o a la página web que desean consultar. Por último, se encuentra el grupo de adultos que tienen un alto nivel de manejo, están interiorizados en el tema y esto les permite, a su vez, tener un control más estricto del uso que hacen los/as niños y niñas de internet, debido a que pueden, por ejemplo, implementar estrategias para bloquear páginas a las que no deseen que accedan. (Barrera Valencia, y Duque Gómez, 2014)

Lo mencionado con anterioridad, desemboca en el hecho de que dependiendo del uso que hacen los adultos de internet, es como esto influye en la dinámica familiar; es decir, cuando no hacen uso del mismo, genera conflictos familiares, debido a la brecha informática que se produce entre ellos y los/as niños y niñas. Mientras que, quienes hacen un uso directo o experto de la red, propician cambios favorables en las interacciones familiares, debido a que se vinculan sobre dudas respecto al uso, o se 
piden ayuda mutua a la hora de acceder a internet. (Barrera Valencia, y Duque Gómez, 2014)

Por otro lado, respecto al uso que hacen los infantes de las tecnologías Sierra (2006) propone que, las/os llamadas/os niñas y niños tecnológicos son aquellos que presentan un conjunto de características novedosas, las cuales mantienen una relación directa con la tecnología y que, como consecuencia, presentan un perfil particularmente orientado hacia las habilidades y destrezas del manejo tecnológico. Por otra parte, Prensky (2010) propone el término "Nativos Digitales" y sostiene que son aquellos/as que han nacido y se han desarrollado utilizando la "lengua digital" de juegos, videos e internet. Estos niños y niñas presentan características tales como: necesidad de recibir información de forma ágil e inmediata, se sienten atraídos por multitareas, les gusta aprender de manera lúdica y no de la manera tradicional. Este autor, también habla de la brecha digital y el desfasaje que se produce entre esta generación y las otras; para explicarlo, acuña el término "Inmigrantes Digitales", refiriéndose a todos aquellos que no han nacido bajo el mundo de las tecnologías, pero sí han hecho un esfuerzo para adaptarse a ellas.

Los niños y las niñas transcurren una gran cantidad de tiempo en interacción con las nuevas tecnologías, lo que las convierte en un potencial medio de socialización (Arza, 2008). A medida que el acceso a los dispositivos electrónicos se extiende, su uso se domestica, se institucionaliza, se formaliza y se confina al ámbito privado (Trinidad y Zlachevsky, 2013). El rol que ocupan los medios de comunicación y los contenidos de las pantallas con las que se vinculan los infantes no es menor, es por ello que la intervención de los adultos es crucial. "La presencia, el compromiso y la atención son las tres claves, ya no sólo de la relación entre los niños, las niñas, los adultos y el juego, sino las llaves para la construcción de vínculos que se enfrenten directamente con las representaciones hegemónicas de los medios de comunicación que, día a día, ubican a los adultos como sujetos prescindibles de la vida de los más chicos (...)" (Duek, 2014, p. 216).

Es por esto, que la problemática planteada nos llevó a preguntarnos, ¿Qué función cumplían las familias en este nuevo contexto tecnológico? En este sentido, les otorgamos un rol primordial a los cuidadores, que son quienes toman las decisiones en relación a la crianza y a la implementación de estrategias para el uso de las tecnologías por parte de los niños y las niñas. Observamos cómo estos, se encuentran atravesados a la hora de elegir la manera de intervenir y tomar decisiones frente al uso de las nuevas tecnologías, las cuales estarán determinadas por el uso que ellos mismos hacen de estas, así como también por las preconcepciones en cuanto a los riesgos y los beneficios que traen aparejadas. En esta misma línea, Carolina Duek y Sebastian Benitez Largui (2018), proponen que, a pesar de las diferencias generacionales, el apropiarse de las tecnologías, constituye un eje central en torno al cual giran los vínculos entre adultos y niñas/os. Las negociaciones, habilitaciones y controles intergeneracionales se configuran en relación con los usos de los dispositivos electrónicos. Los autores sostienen que el saber hacer facilita, y simultáneamente, tensiona estos vínculos.

Como ya sabemos, la familia es uno de los principales agentes de socialización en edades tempranas. En esta etapa, los/as niños/as son moldeables y comienzan a formarse a partir de la observación y de la imitación a los adultos. Estos mismos, son modelos que los niños y las niñas imitan y van inhibiendo o estimulando determinadas conductas (Froufe, 1995). En este sentido, cuando los adultos a cargo se relacionan 
con los/as infantes, se ponen en juego estrategias de socialización, pretendiendo regular la conducta de los niños y las niñas en dirección a lo que estos desean de acuerdo a su personalidad (Córdoba, 2014). Es decir, las Estrategias de Socialización, son todas aquellas conductas que los adultos conciben como apropiadas y deseables tanto para el desarrollo, como para la integración social de niños y niñas. Así como también, los medios de los que disponen estos adultos para alcanzar dichos estados esperables. (Arámbula-Román et al., 2012, en Córdoba, 2014)

Asimismo, las familias operan a través de Pautas Transaccionales que establecen -de qué manera, cuándo y con quién relacionarse-, y van a ser estas las que afiancen al sistema. Es decir, dichas pautas regulan la conducta de los/as integrantes de la familia, y a su vez son mantenidas por dos sistemas de coacción; uno genérico, que son las reglas universales que guían la organización familiar, y otro formado por reglas idiosincráticas, que implican las expectativas mutuas de los diferentes miembros de la familia. A pesar de esto, la estructura familiar debe poseer la capacidad de adaptación a los cambios, es decir, la flexibilidad para movilizar y readaptar las pautas transaccionales cuando se lo considere necesario, para la funcionalidad del sistema. (Minuchin, 1982)

Por otro lado, como ya hemos mencionado, la incorporación de las tecnologías en la trama familiar, produjo una variedad de cambios en cuanto a los estilos comunicativos entre cuidadores, niños y niñas. Esto a su vez puede ser percibido de diferentes maneras, atribuyéndole características, tanto negativas como positivas, que devienen del uso que cada uno haga de las mismas (Barrera Valencia, y Duque Gómez, 2014). Es decir, el uso de las tecnologías en las infancias, no puede considerarse como directamente perjudicial, pero tampoco exclusivamente positivo. (Yubero, Larrañaga, Navarro, y Elche, 2018)

En cuanto a los riesgos o conflictos que trae aparejado el uso de tecnologías, podemos mencionar, que tanto adultos como infantes se desresponsabilizan de sus tareas por estar conectados a Internet. El mayor conflicto señalado por parte de los adultos es que los/as niños/as no realizan los deberes y no colaboran en mantener el orden del hogar por estar con las pantallas. Además, el libre acceso a la red, impide que los adultos puedan controlar a quienes los infantes dejan ingresar en la vida familiar, con quienes se contactan y que comparten con el resto. En consonancia con esto, es que también las relaciones entre los miembros de la familia pueden volverse superficiales y frías, debido a que niños y niñas prefieren estar conectados a internet, antes que compartiendo con su familia, y esto genera el debilitamiento de los vínculos familiares. (Barrera Valencia, y Duque Gómez, 2014)

Es en relación a esto, que podríamos clasificar los riesgos en dos grupos, los que son específicos del uso de internet, y otros ligados directamente a los servicios utilizados en la red; siendo más vulnerables a correr estos riesgos, aquellos niños/as y adolescentes que presentan conflictos familiares, baja autoestima, búsqueda de afecto y atención o antecedentes de violencia familiar o depresión. (García Piña, 2008)

Por otro lado, también podemos situar familias que destacan aspectos positivos sobre el uso del internet y las pantallas, estas mencionan que las relaciones familiares se vieron beneficiadas ya que comparten más tiempo al enseñarles a usar los dispositivos o internet, generando un acercamiento de ambas partes (Barrera Valencia, y Duque Gómez, 2014). En adición a esto, podemos situar otros beneficios ligados al hecho de que hoy en día las nuevas generaciones, al haber nacido en la era tecnológica, hacen uso de los dispositivos para actividades tanto de 
entretenimiento como escolares; desarrollando de este modo, habilidades comunicacionales interactivas y simbólicas, que los llevan a incorporar un lenguaje de símbolos comunes, rompiendo barreras culturales. (Domínguez Fernández, Martínez Jaén, y Ceballos García, 2017)

Es por todo lo anteriormente mencionado, que entendemos a la incorporación de las tecnologías en las familias como un proceso dinámico e influenciado por múltiples variables, así como también, por la cotidianeidad y las creencias de cada sistema en particular.

\section{Materiales y método}

La presente investigación, se realizó en el año 2019 en la Ciudad de Córdoba, Argentina; y como ya se mencionó, tuvo por objetivo conocer el uso de tecnologías en niños y niñas de entre 4 y 12 años de edad, respecto a las pautas transaccionales y las estrategias de socialización implementadas por las familias.

Se llevó a cabo con un diseño no experimental, aquel en el cual el investigador no manipula las variables a investigar, sino que estas son observadas tal cual suceden en su ambiente natural para luego ser analizadas (Hernández Sampieri, Fernández Collado, y Baptista Lucio, 2010). El diseño fue de tipo transversal, entendiendo a éste como aquel que tiene el propósito de recolectar datos en un momento y un periodo de tiempo determinado. El objetivo del mismo es describir y analizar las relaciones que se presentan entre las diferentes variables en un momento dado.

En cuanto al tipo de estudio, lo clasificamos como mixto, debido a que busca una integración o vinculación entre lo cualitativo y lo cuantitativo, logrando el mayor grado de combinación entre dichos enfoques. Una de las ventajas del enfoque mixto, es que brinda una perspectiva más precisa del fenómeno a estudiar, ya que la percepción del mismo es más integral y completa (Hernández Sampieri et al., 2010). La población que se estudió corresponde a personas a cargo (padres, madres, tutores) de niños y niñas de entre 4 y 12 años de edad.

A la hora de realizar la recolección de datos, utilizamos dos tipos de instrumentos: una entrevista semiestructurada y un cuestionario online construido "ad hoc". La muestra estuvo conformada por 165 personas que cumplieron con los requisitos anteriormente mencionados, y específicamente, quienes completaron el cuestionario online; siendo de este modo, una muestra aleatoria. En cuanto a la entrevista, participaron 10 adultos a cargo de niños y niñas de entre 4 y 12 años de edad; siendo de esta manera, una muestra de tipo no probabilística debido a que, "la elección de los elementos no depende de la probabilidad, sino de causas relacionadas con las características de la investigación o los propósitos del investigador" (Johnson, 2014, Hernández-Sampieri et al., 2013 y Battaglia, 2008, en Sampieri et al., 2010, p.176). Es decir, al seleccionar los elementos que lo componen, no se cumple el requisito de aleatoriedad y como consecuencia, los resultados no pueden ser generalizados más allá de los casos observados. (Romero, Bologna, 2013)

Por su parte, el muestreo que se utilizó fue el "de juicio o intencional", conocido también como "selección experta". El investigador es quien decide qué elementos son los más adecuados a los fines de su investigación. Los participantes fueron seleccionados según su representatividad para la investigación, con el objetivo de 
profundizar el análisis a partir del testimonio de algunos de ellos/as (Romero, Bologna, 2013).

Para cumplir con los objetivos propuestos en la investigación, se llevó a cabo una prueba piloto del cuestionario, en la que participaron cinco personas; con el objetivo de realizar ajustes en los ítems a responder y atendiendo a la adecuada comprensión por parte de los participantes. A partir de la recolección de datos obtenidos, pudimos observar ciertas preguntas que presentaron dificultades a la hora de ser respondidas o que serían un obstáculo al momento del análisis. De esta forma, se readaptó el instrumento para una mayor precisión y exactitud de la información obtenida.

Por último, para el análisis de datos cuantitativo, en primer lugar, se seleccionó el software SPSS 23; se procedió a la carga de los datos, ingresando las 165 respuestas obtenidas para cada indicador de las diferentes variables a analizar. Luego de eso, se identificó, en qué variables se presentaron casos perdidos, y esto fue tenido en cuenta al realizar el análisis. Se realizó un análisis de frecuencia para cada indicador, del cual obtuvimos los porcentajes totales y el número de casos para cada pregunta.

En cuanto al análisis cualitativo de la entrevista, se realizó agrupando los datos en base a las tres categorías conceptuales estudiadas (pautas transaccionales, estrategias de socialización y uso de tecnologías). Se buscaron indicadores que referían a las mismas y se incluyeron en las diferentes preguntas y luego se relacionó cada respuesta con su respectiva categoría.

\section{Resultados}

Análisis de datos Cualitativo

Categoría Pautas transaccionales

Para la categoría Pautas transaccionales, se pudo observar, en base a las respuestas obtenidas de las entrevistas realizadas, que en el indicador "Regulación del uso de las tecnologías como pauta en el hogar", un $40 \%$ de la muestra respondió que no tenían horarios estipulados para el uso de tecnologías en los/as niños/as, mencionando que los/as infantes decidían libremente cuándo utilizar las pantallas y cuándo dejarlas. Mientras que un $20 \%$, manifestó que los horarios no eran estables, iban variando dependiendo el día y las actividades que quieran realizar; el restante $40 \%$, señaló que los/as niños/as utilizaban las pantallas en horarios determinados, asociados en su mayoría a la alimentación, es decir, mientras desayunaban, merendaban o antes de cenar, mencionando que era "un rato tecnología" y "otro rato diferentes actividades". Del total de la muestra, solo un adulto respondió que el niño no utilizaba las tecnologías todos los días, y dos aludieron a que los fines de semana el uso se incrementaba. Observando la regulación del uso de las tecnologías como pauta en el hogar, se obtuvieron datos en cuanto a la manera y los momentos en que los infantes interaccionan con las pantallas, y si esto se da de manera libre o regulada por los adultos; hablándonos probablemente de las reglas idiosincráticas que se manifiestan en dicho sistema familiar y de si se cumplen las expectativas tanto de adultos como de niños y niñas en cuanto a cuando usar y cuando no las tecnologías. (Minuchin, 1982) 
Por otro lado, para el indicador "Comportamiento o conductas del niño/a como determinante del uso de las tecnologías", observamos que un $40 \%$ de la muestra mencionó que el comportamiento del niño/a no determinaba el uso de pantallas, es decir, las mismas no eran prohibidas como modo de castigo o penitencia. En relación a esto, una de las entrevistadas manifestó que intentó la estrategia de la prohibición de las tecnologías como castigo, pero que no funcionó, que en el caso de su familia no servía esa modalidad. En contraposición, tenemos el mismo porcentaje de entrevistados/as, es decir, otro $40 \%$ que señaló que la buena o mala conducta del niño/a, sí determinaba el uso de pantallas. El restante $20 \%$ de la muestra, aludió a que a veces el uso de las tecnologías estaba condicionado al comportamiento y a veces no, es decir, que no siempre la consecuencia era dejar sin tecnologías al niño/a.

En cuanto al siguiente indicador, a saber, "Acuerdos respecto al uso de pantallas", obtuvimos un $40 \%$ de los/as entrevistados/as que señalaron no tener acuerdos respecto al uso de tecnologías por parte de los/as niños/as, ya sea, porque sostenían que no hace falta o porque al momento de dejar de usar las tecnologías, alcanzaba con decirles que se acabó su tiempo. En otro $30 \%$ de las respuestas, observamos que eran familias que sí tenían acuerdos más específicos y que estos consistían en explicarles al inicio de su uso, en qué momentos hacerlo y en cuáles no, o en señalarles cuando ya era hora de dejar las pantallas. Mientras que un $20 \%$ de la muestra, señaló tener un estilo más democrático, es decir, las pautas se charlaban y acordaban entre todos/as, o se iban decidiendo sobre la marcha. Y por último, un 10\% de la muestra, mencionó ser "quien pone las reglas", teniendo el/la niño/a que obedecer, es decir, formaría parte de quienes sí tenían ciertas reglas, pero estas no eran acordadas de manera conjunta.

Por último, los resultados obtenidos de las observaciones del indicador "Modalidad adoptada por los adultos para el establecimiento de límites en cuanto a las tecnologías", demostraron que la mitad de la muestra elegía como estrategia de establecimiento de límites, el dialogar con los/as niños/as, explicándoles y enseñándoles, tanto lo positivo como lo negativo de las tecnologías, y una de las entrevistadas manifestó que de esta manera obtenía mejores resultados y comprensión por parte del niño/a. Otro $30 \%$ de la muestra, realizaba lo contrario, es decir, no le daba demasiadas explicaciones a los/as niños/as, directamente le sacaban el celular o aparato tecnológico de las manos. El $20 \%$ restante de los/as entrevistados/as, en este indicador, mencionó que le iban avisando a los/as niños/as para que finalicen el uso de pantallas, pero además intentaban generar interés en otras actividades compartiendo tiempo con ellos/as.

En el gráfico a continuación, se representan las respuestas que se obtuvieron en mayor porcentaje para los diferentes indicadores a analizar para la Variable Pautas Transaccionales. 


\section{Gráfico 1: Pautas Transaccionales}

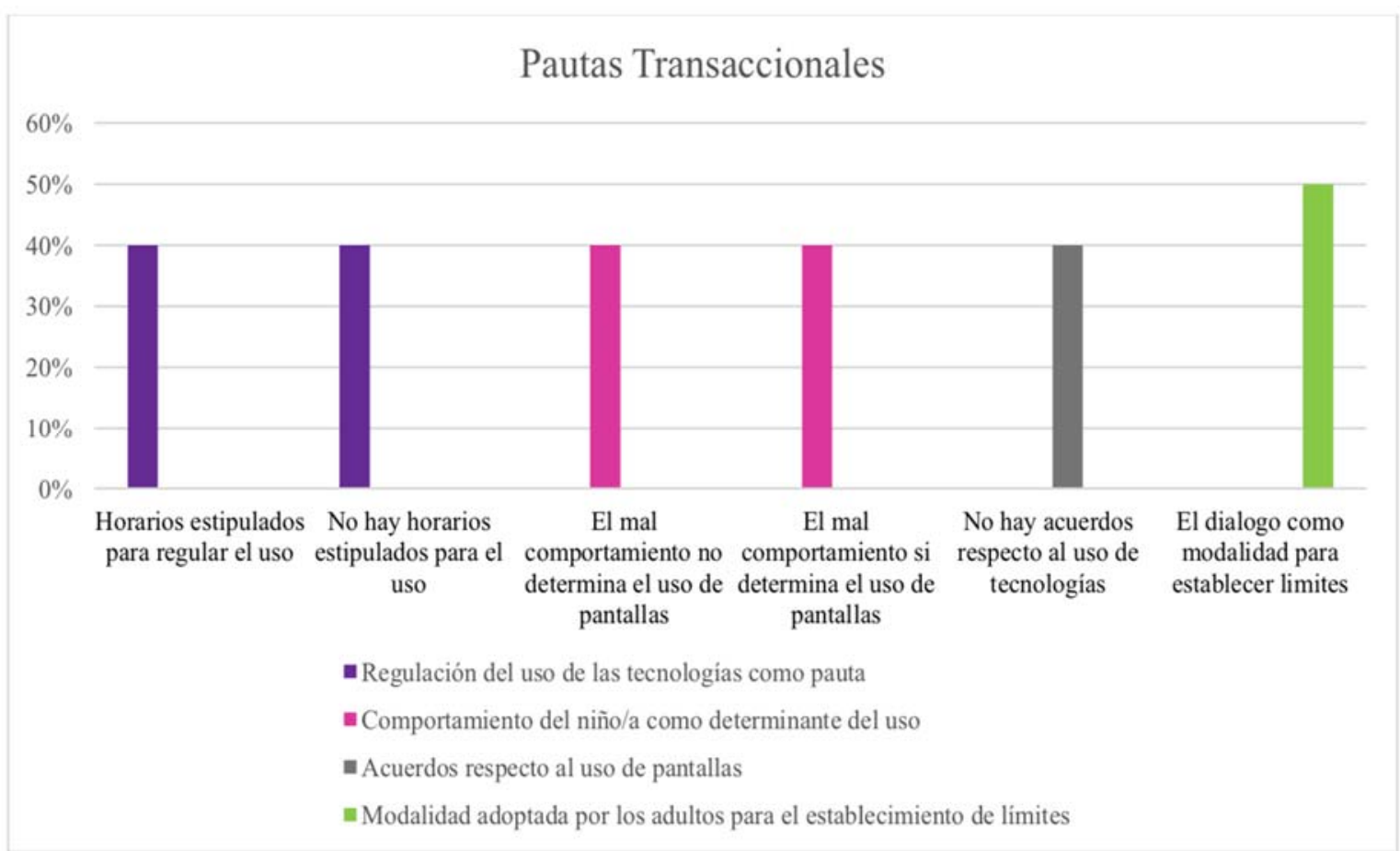

Fuente: elaboración propia

Categoría Estrategias de socialización

Con respecto a la categoría Estrategias de socialización, fue posible visualizar que en el indicador "Concepciones de los adultos sobre el uso de tecnologías por parte de los/as niños/as", el $80 \%$ de los/as entrevistados/as consideró apropiado que los/as infantes utilicen las tecnologías, sosteniendo que son una herramienta útil que forma parte de la época en la que viven, y que el acceso a las mismas les brinda múltiples posibilidades. Sin embargo, mencionaron estar de acuerdo siempre que su uso tenga limitaciones, en una medida no excesiva y con supervisión de un adulto. Por otro lado, un $20 \%$ de los/as entrevistados/as respondió que, a pesar de no estar de acuerdo, era inevitable que esto suceda, debido al contexto actual en el que están inmersos los/as infantes. Siguiendo esta línea, Iriarte Díazgranados (2011), propone que la virtualidad configura un nuevo entorno que es imposible de evitar.

Para el indicador "Creencias de los adultos en cuanto al uso apropiado de aparatos tecnológicos", un $40 \%$ de los cuidadores respondió que consideraba que lo más conveniente era que las/os niñas/os utilizaran las tecnologías con fines académicos, donde pudiesen investigar, aprender e informarse. Por otro lado, un $30 \%$, mencionó que lo más apropiado era usar los aparatos tecnológicos sin abusar de ellos, realizando un uso razonable y con buenos objetivos. Con respecto al $30 \%$ restante de los/as entrevistados/as, sostuvieron que consideraban apropiada la utilización de las tecnologías a los fines de entretenimiento, preferentemente, viendo contenido acorde a las edades e intereses de cada niño/a. De la totalidad de la muestra, pudimos 
observar que un $80 \%$ de las creencias de los adultos en cuanto al uso apropiado de las tecnologías, se correspondía con lo que sucedía en sus familias, lo cual denotó que estas creencias funcionaban como estrategias de socialización, regulando la conducta de los/as niños/as en función de lo que los adultos deseaban. El $20 \%$ restante manifestó que el uso que le daban los/las niños a las tecnologías, no era lo esperable para ellos/as.

En el indicador "Ausencia del uso por parte de los adultos como estrategia", a partir de las respuestas obtenidas, pudo observarse que la mitad de los/las entrevistados/as manifestaron que en ningún momento del día dejaban de usar los aparatos tecnológicos como estrategia para que los/as infantes no hagan uso de estos. Con respecto a la otra mitad, sostuvieron que sí dejaban de usarlos frente a los/as niños/as, mencionando utilizar como estrategia el silenciar los celulares y sólo responder a mensajes de urgencia, o no usar dispositivos electrónicos en las comidas familiares o en aquellos momentos compartidos. Sin embargo, del total de los adultos entrevistados/as, uno manifestó haber intentado con anterioridad, dejar de usar los celulares en la mesa para poder conversar, pero esta estrategia no dio resultados en su familia. También una de las entrevistadas aludió a que a veces, intentaba no utilizar el celular delante de su hija, y que cuando lo hacía la niña le reclamaba diciendo que estaba "todo el día" en las redes. En la temprana infancia, los infantes toman como modelo de referencia a sus adultos significativos, lo que conlleva a que imiten su conducta (Froufe, 1995). Lo cual permite entrever que aquellas familias que respondieron no tener como estrategia el dejar de usar las tecnologías frente a los/as niños/as, no están teniendo en cuenta la relevancia de su conducta y que la misma influye en el ulterior desarrollo del niño o la niña.

Por último, en el indicador "Modalidad de regulación del tiempo de uso en niños/as", a partir de las respuestas obtenidas, pudo observarse que un $40 \%$ de la muestra, expresó que estipulaban horarios como estrategia para regular el uso de las tecnologías. Por otro lado, un $20 \%$ de los/as entrevistados/as afirmó que no tenían estrategias específicas y que en sus hogares el/la niño/a usaba los dispositivos electrónicos libremente. En cuanto al $40 \%$ restante, pudo observarse que las estrategias que utilizaban los adultos para regular el uso de las tecnologías en los/as niños/as eran múltiples y variadas; esta tarea es multifacética y depende de las circunstancias del entorno y la diversidad de demandas que los/as niños/as plantean (Córdoba, 2014). Esto se pudo visualizar cuando algunos entrevistados/as mencionaron que utilizaban como estrategia la "manipulación", aludiendo a que el/la niño/a, para poder usar las tecnologías, primero debería cumplir con sus deberes y tareas diarias, otros afirmaron, que regulando su propio uso de las pantallas, lograban regular el de los/as infantes; también mencionaron que proponían actividades alternativas como "en los días soleados se realizan actividades al aire libre", "cuando hay amigos en la casa se comparten otros juegos", "en la hora del almuerzo hay que mirarse a la cara y charlar", entre otras.

En el siguiente gráfico, se representan las respuestas que se obtuvieron en mayor porcentaje para los diferentes indicadores a analizar de la Variable Estrategias de Socialización. 


\section{Gráfico 2: Estrategias de socialización}

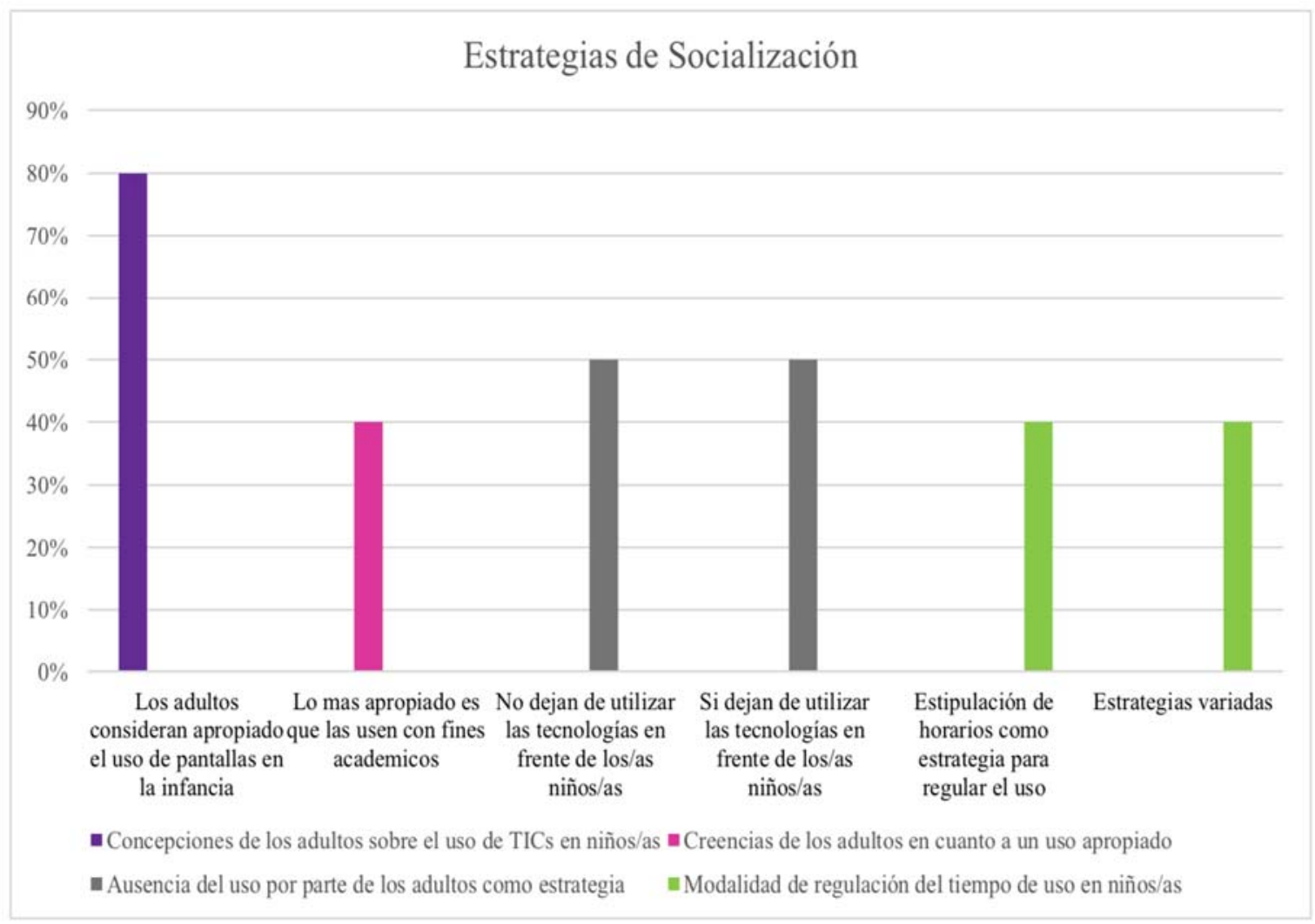

Fuente: elaboración propia

Categoría Uso de tecnologías

Para la categoría Uso de tecnologías, se pudo observar, a partir de las respuestas obtenidas de las entrevistas realizadas, que en el indicador "Frecuencia del uso de las tecnologías en niños y niñas", un $40 \%$ de la muestra afirmó que los/as niños/as hacían uso de las tecnologías 3 horas todos los días, otro $40 \%$ denotó 2 horas por día, todos los días y el $20 \%$ restante, refirió a un menor uso (un $10 \%$, una hora por día únicamente, y otro $10 \%$, utilizaba las tecnologías de lunes a viernes, solo tres días). Generalmente el uso se daba al regreso de la escuela, por la tarde.

Por otra parte, para el indicador "Edad de iniciación del uso", se observó que el $50 \%$ de la muestra, indicó que la edad de iniciación del uso de las tecnologías se dio a los meses o al año de vida. El restante 50\%, mencionó edades desde los 2 hasta los 5 años; dentro del cual, un 10\% indicó a los 2 años, un $10 \%$ a los 3, un $20 \%$ a los 4 y un $10 \%$ a los 5 años de edad.

Respecto al siguiente indicador, "Finalidades del uso por parte de los/as niños/as", el $100 \%$ de la muestra respondió que, principalmente, la finalidad del uso era el entretenimiento. Sin embargo, un $40 \%$ afirmó que, en menor medida, también utilizaban las tecnologías con fines académicos. Además, otro $30 \%$ mencionó, que los/as niños/as la utilizaban para buscar información respecto a inquietudes. Solo un entrevistado, afirmó que el uso se debía exclusivamente a los fines de entretenimiento, 
y otro, añadió que consideraba que se debía a la comodidad de los/as padres/madres. Solo un $10 \%$ agregó la comunicación. Como mencionamos anteriormente, el uso de tecnologías, suele tener como finalidad realizar actividades académicas, de entretenimiento y ocio o de comunicación interpersonal y además, este uso se caracteriza por el concepto de interactividad, ya que permite la comunicación entre usuarios (Vodopivec y Samec, 2013). Tomando esta definición, las finalidades del uso coinciden con las respuestas de los/as entrevistados/as, en cuanto al entretenimiento y lo académico. Sin embargo, en este caso, la utilización de tecnología a los fines de comunicación es escasa; hipotetizamos que esto se debe a que se trata de menores de 12 años y aún no es una actividad de gran interés. En otras palabras, las principales actividades que realizan en internet son prácticas lúdicas. El juego es un espacio en el que los/as niños/as incorporan reglas y pautas sociales, es decir, también es una instancia de aprendizaje y socialización. (Trinidad y Zlachevsky, 2013)

Según el indicador "Frecuencia y finalidad del uso por parte de los adultos", se puso en evidencia que el total de la muestra hacía uso de las tecnologías con el objetivo de entretenimiento. Sin embargo, de ese $100 \%$, un $70 \%$ lo hacía, principalmente, con fines laborales y en menor medida, para entretenimiento. Un $10 \%$, aclaró que además de utilizar las tecnologías para trabajar y entretenerse, las utilizaba para informarse. Otro $20 \%$, para entretenerse y comunicarse, y no para trabajar. Solo un $10 \%$, expresó usarlas, con el único objetivo de trabajo. En este sentido, los adultos presentaban un alto nivel de manejo de la tecnología. Retomando los aportes de los autores estudiados, se puede afirmar que esto les permitiría tener un control más riguroso del uso que hacen los niños y las niñas de internet. A su vez, pueden compartir y cooperar con los/as infantes en actividades en torno a la tecnología, propiciando cambios favorables en las interacciones familiares (Barrera Valencia, y Duque Gómez, 2014).

Para el indicador "Utilización de las tecnologías por parte de los adultos en presencia de los/as niños/as.", el 70\% contestó que hacía uso de las tecnologías en presencia de los/as infantes; de ese total, un $10 \%$ expresó que lo hacía en presencia porque compartía con su hijo/a esta actividad. A su vez, un $20 \%$ contestó "a veces", y un $10 \%$, "intento que no". En otras palabras, el total de la muestra en algún momento estuvo en contacto con las tecnologías en presencia de los/las niños/as.

Por último, a partir de lo observado en el indicador "Conflictos en el ámbito familiar en función del uso de tecnologías", se pudo aseverar que el total, el $100 \%$ de la muestra, consideraba que el uso de las tecnologías ha generado algún conflicto o discusión en el ámbito familiar. Los motivos variaron acorde a cada familia, pero se han hallado algunas coincidencias generales. El $40 \%$ de la muestra, expresó que los conflictos se debían al uso de tecnologías en horarios de encuentro familiar, los cuales solían ser en la cena, siendo esta el momento donde se reúnen, conversan, comparten, etc. A su vez, otra porción de la muestra, conformada por un $20 \%$, relató conflictos dados por el uso de redes sociales, páginas o aplicaciones, por parte de los/as infantes, que los adultos consideraban inapropiadas para su edad. Mientras tanto, tan solo un $20 \%$, aludió a que los conflictos se debían al uso excesivo de las tecnologías. En cuanto al $20 \%$ restante de la muestra, estuvo conformado por un $10 \%$ que indicó haber tenido conflictos, pero ya no al momento de la entrevista, ya que se habían ordenado y estipulado los tiempos y momentos de uso, y el otro $10 \%$, ha respondido "sí", sin explicitar los motivos. 
En el gráfico que se presenta a continuación, se representan las respuestas que se obtuvieron en mayor porcentaje para los diferentes indicadores a analizar de la Variable Uso de Tecnologías.

\section{Gráfico 3: Uso de Tecnologías}

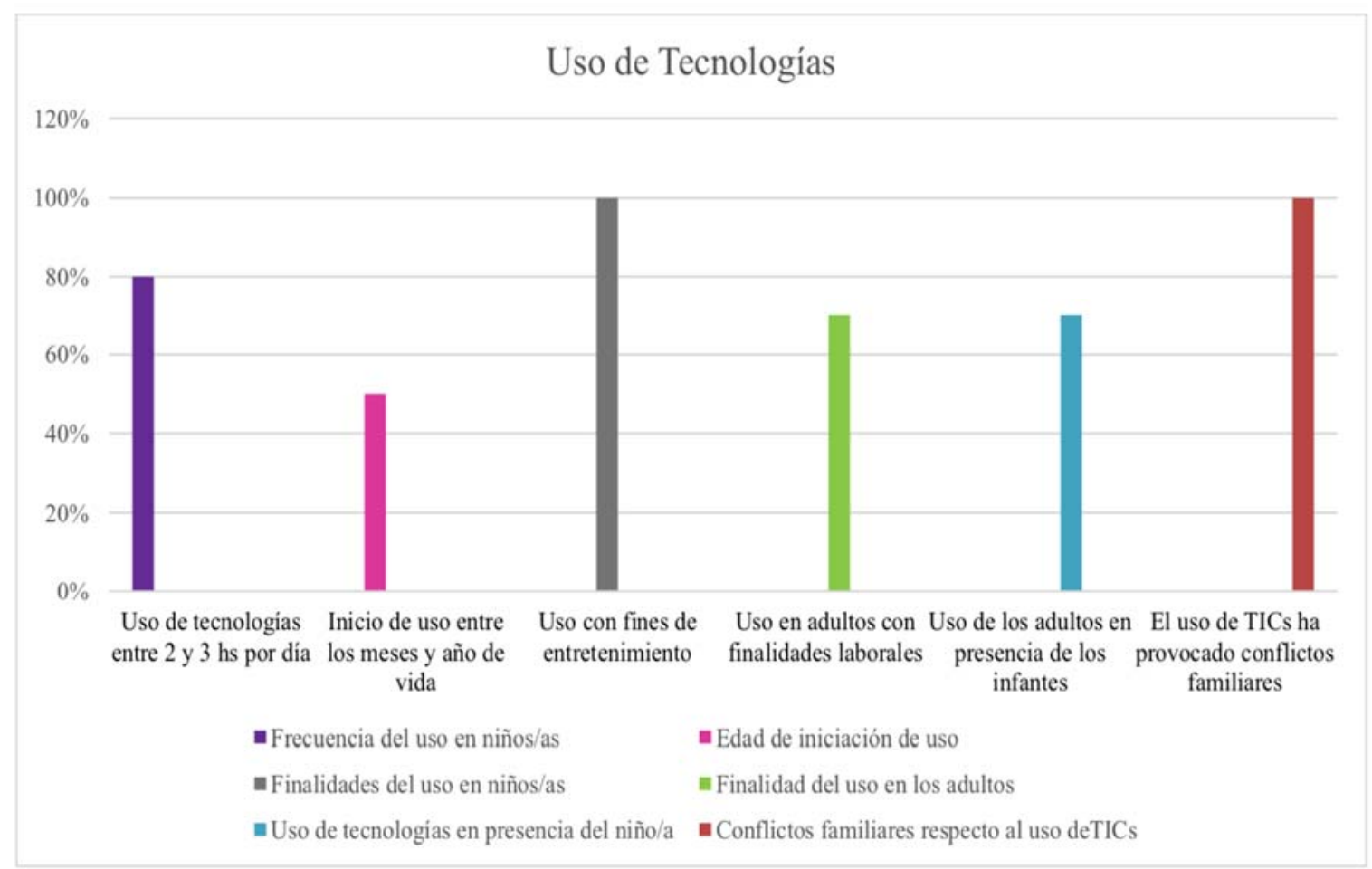

Fuente: elaboración propia

Análisis de datos cuantitativo

A continuación, se presentan las tablas $1,2,3$ y 4 que reflejan los datos sociodemográficos y los de las categorías Estrategias de Socialización, Pautas Transaccionales y Uso de Tecnologías, respectivamente. 
Tabla 1. Descripción de la muestra

\begin{tabular}{|c|c|c|c|c|c|c|}
\hline & Frecuencia & Porcentaje & Media & Moda & $\begin{array}{c}\text { Desv } \\
\text { Est }\end{array}$ & Rangc \\
\hline $\begin{array}{l}\text { Edad del } \\
\text { Entrevistado/a }\end{array}$ & - & - & 37,95 & 39 & 7,847 & $21-66$ \\
\hline \multicolumn{7}{|l|}{$\begin{array}{l}\text { Género del } \\
\text { entrevistado }\end{array}$} \\
\hline Masculino & 27 & 16,4 & - & - & - & - \\
\hline Femenino & 138 & 83,6 & - & - & - & - \\
\hline \multicolumn{7}{|l|}{ Nivel máximo de estudios } \\
\hline Primario completo & 1 & 0,6 & - & - & - & - \\
\hline Secundario incompleto & 3 & 1,8 & - & - & - & - \\
\hline Secundario completo & 30 & 18,2 & - & - & - & - \\
\hline Terciario incompleto & 9 & 5,5 & - & - & - & - \\
\hline Terciario completo & 19 & 11,5 & - & - & - & - \\
\hline Universitario incompleto & 31 & 18,8 & - & - & - & - \\
\hline Universitario completo & 72 & 43,6 & - & - & - & - \\
\hline
\end{tabular}

Fuente: elaboración propia

En la Tabla 1 podemos ver que un dato significativo es el gran porcentaje de participantes del género femenino con respecto al masculino.

Tabla 2. Estrategias de Socialización

\begin{tabular}{lccc}
\hline & Frecuencia & Porcentaje & Moda \\
\hline $\begin{array}{l}\text { ¿Qué hago cuando salgo y el infante está } \\
\text { inquieto? }\end{array}$ & - & - & 2 \\
Le presto mi celular & 50 & 30,3 & - \\
Elijo un lugar que cuente con espacio de juegos & 76 & 46,1 & - \\
Me levanto de la mesa y voy a jugar él/ella. & 21 & 12,7 & - \\
Lo/la reto para que se quede sentado/a & 18 & 10,9 & - \\
Ocupaciones luego del horario laboral & - & - & 2 \\
Sigo con mi trabajo & 5 & 3 & - \\
Ayudo al niño/a a hacer la tarea & 60 & 36,4 & - \\
Realizo otras actividades recreativas & 22 & 13,3 & - \\
Aprovecho para jugar con el/la niño/a & 50 & 30,3 & - \\
Otra & 28 & 17 & 2 \\
Regulación del tiempo de uso de pantallas & - & - & - \\
No lo regulo & 22 & 13,3 & - \\
Tenemos pautados horarios determinamos & 74 & 44,8 & - \\
Sola las usa cuando finaliza sus tareas & 46 & 27,9 & - \\
Otra & 23 & 13,9 & -
\end{tabular}

Fuente: elaboración propia 
Un resultado significativo a los fines de la investigación fue que la estrategia por excelencia elegida por los adultos para regular el tiempo de uso de las tecnologías, era el establecimiento de horarios determinados.

Tabla 3: Pautas Transaccionales

\section{Frecuencia Porcentaje Moda}

\begin{tabular}{|c|c|c|c|}
\hline Momentos en que utiliza las tecnologías & - & - & 4 \\
\hline Cuando lo desea & 45 & 27,3 & - \\
\hline Cuando estoy ocupado/a & 19 & 11,5 & - \\
\hline Cuando se porta bien & 17 & 10,3 & - \\
\hline En los horarios estipulados & 84 & 50,9 & - \\
\hline Acuerdos respecto al uso de tecnologías & - & - & 2 \\
\hline Si, son negociados entre todos & 52 & 31,5 & - \\
\hline $\mathrm{Si}$, los decidimos los adultos & 81 & 49,1 & - \\
\hline No, no hay acuerdos & 21 & 12,7 & - \\
\hline No, usa las pantallas libremente & 11 & 6,7 & - \\
\hline Sostenimiento del NO en el tiempo & - & - & 4 \\
\hline Nunca & 0 & 0 & - \\
\hline Casi nunca & 2 & 1,2 & - \\
\hline A veces & 31 & 18,8 & - \\
\hline Frecuentemente & 78 & 47,3 & - \\
\hline Siempre & 54 & 32,7 & - \\
\hline Reacción del infante frente al NO & - & - & 2 \\
\hline Lo acepta sin cuestionar & 24 & 14,5 & - \\
\hline Lo acepta pero pide explicaciones & 79 & 47,9 & - \\
\hline No lo acepta y se enoja & 40 & 24,2 & - \\
\hline Llora y pide explicaciones & 18 & 10,9 & - \\
\hline Otra & 4 & 2,4 & - \\
\hline Control de los hábitos respecto al uso & & & 2 \\
\hline Si, siempre & 74 & 44,8 & - \\
\hline $\mathrm{Si}$, cuando estoy en el hogar & 87 & 52,7 & - \\
\hline No, no lo considero necesario & 4 & 2,4 & - \\
\hline No, no me corresponde controlarlos & 0 & 0 & - \\
\hline
\end{tabular}

Fuente: elaboración propia 
Tabla 4: Uso de Tecnologías

\begin{tabular}{rccccc}
\hline & Frecuencia & Porcentaje & Media & Moda & $\begin{array}{c}\text { Desv } \\
\text { Est }\end{array}$ \\
\hline Frecuencia de acceso & - & - & - & 4 & - \\
Nunca & 0 & 0 & - & - & - \\
Una vez a la semana & 12 & 7,3 & - & - & - \\
Varios días a la semana & 40 & 24,2 & - & - & - \\
Todos los días & 113 & 68,5 & - & - & - \\
Edad de comienzo & - & - & 4,14 & 2 & 2,227 \\
Finalidad* & & & & & \\
Entretenimiento & 161 & 97,6 & - & - & - \\
Comunicación & 64 & 38,8 & - & - & - \\
Académico & 63 & 38,2 & - & - & - \\
Otros & 8 & 4,8 & - & - & - \\
\hline
\end{tabular}

Fuente: elaboración propia

*Los datos recogidos en base a las diferentes finalidades no son excluyente, es decir, los/as encuestados/as tenían la posibilidad de marcar más de una opción.

Cabe destacar que el $68,5 \%$ de la muestra señaló que los/as infantes utilizaban las tecnologías todos los días, siendo este un dato significativo; así como también el hecho de que el $97,6 \%$ las usaba con fines de entretenimiento.

\section{Discusión}

La infancia es un período clave para el posterior desarrollo, por lo que resulta importante atender al uso que hacen los infantes de las tecnologías. Consideramos que es tarea de los adultos, intentar reducir la cantidad de horas al día en que los/as infantes permanecen frente a una pantalla, para favorecer un uso más saludable.

En la Categoría Pautas Transaccionales, pudimos obtener datos en cuanto a la manera y a los momentos en que los/as infantes interaccionaban con las pantallas. Además, se observó si esto se daba de manera libre o regulada por los adultos; informándonos sobre las reglas que se manifiestan en cada sistema familiar; tanto idiosincráticas como universales. Sumado a esto, se atendió a si se cumplían las expectativas de los adultos y de los de niños y niñas en cuanto a los momentos destinados a usar las tecnologías. Se puso en evidencia que las reglas funcionaban de guía para la organización familiar y a su vez, regulaban la conducta de los integrantes de la familia. Por ejemplo, una gran mayoría manifestó que, a nivel familiar contaban con reglas universales establecidas: ante un comportamiento inapropiado de los/as infantes, la consecuencia era prohibirles aquellas actividades de recreación, incluidas las tecnologías. Otros adultos, por ejemplo, expresaron que controlaban los hábitos del niño/a con respecto al uso de tecnologías, pero lo hacían sólo en el hogar. Esto nos brinda información de que al no poder controlar lo que los/as niños/as hacen 
con las tecnologías cuando los adultos no están presentes, se genera una brecha en la cual los/as infantes podrían tener acceso a contenido no apropiado o hacer un uso desmedido.

Por otro lado, en base a los resultados, se vislumbra que los límites se suelen establecer de manera acordada y dialogando con los/as niños/as. Esto se debe a que la mitad de los/as entrevistados/as manifestó que tiene la capacidad de flexibilizar y readaptar las pautas transaccionales, a medida que el sistema lo demande o lo necesite, para mayor funcionalidad. Esto no sólo demuestra que probablemente sean estilos parentales de tipo democrático (Diana Baumrind, en Remache Delgado, 2014), sino que, además, les brinda mayores herramientas a los/as niños/as, para entender el porqué de las reglas establecidas por los adultos y percibirlo como un beneficio para ellos/as mismos/as (y no como una restricción impuesta unilateralmente por los mayores).

Es destacable que, a pesar de que un porcentaje de la muestra alude a que no tenía acuerdos ni pautas específicas respecto al uso de las pantallas, éstas sí existían. Cabe aclarar que en la mayoría de los casos se expresó no contar con acuerdos ni pautas explícitas, pero, igualmente, se observó que sí estaban presentes, sin que los adultos sepan que funcionan como tales. Es importante reflexionar sobre el papel fundamental de los adultos, guiando y acompañando el uso de las tecnologías; siendo más efectivo que los acuerdos, sean acordados, valga la redundancia. Es decir, que se establezcan en conjunto, para que los mismos infantes, poco a poco, autorregulen y autogestionen el uso, a través de la incorporación y del aprendizaje de las reglas o pautas dispuestas en el hogar. A su vez, consideramos conveniente, que las mismas sean claras y consistentes. Es decir, que no sean contradictorias ni ambiguas, y que además, se sostengan en el tiempo. En relación a esto último, se evidencia que los adultos presentaron cierta labilidad o flexibilidad a la hora de poner límites respecto al uso de tecnologías, ya que las decisiones no suelen ser sostenidas a lo largo del tiempo. Esto, en alguna medida, resulta perjudicial para los infantes ya que reciben "dobles mensajes".

Respecto a la Categoría Estrategias de Socialización, obtuvimos que la estrategia de socialización que predominaba y consideraban más eficaz las familias, era la de pautar horarios para regular el uso de tecnologías. Además, las respuestas recolectadas, nos permitieron dar cuenta de cierta contradicción en los adultos. Por un lado, eran conscientes de estar inmersos en un mundo en permanente movimiento y que la virtualidad configura un nuevo entorno que es imposible de evitar (Iriarte Díazgranados, 2011); pero, por otro lado, también consideraron que son ellos, quienes tienen que regular el uso, para que este no sea desmedido y así disminuir posibles riesgos. En esta línea, sabemos que las conductas de los adultos cumplen un rol crucial en la construcción de subjetividad de los/as niños/as, así como también en el desarrollo de su aprendizaje. En la temprana infancia, estos/as infantes, toman como modelo de referencia a estos adultos significativos, lo que conlleva a que imiten su conducta (Froufe, 1995). Como ya mencionamos, esto nos lleva a pensar que aquellas familias que, por ejemplo, respondieron no tener como estrategia el dejar de usar las tecnologías frente a los/as niños/as, no estaban vislumbrando la importancia de su conducta, siendo que la misma podía ser imitada por el niño o la niña.

Además, nos parece relevante destacar que a lo largo de la toma de las entrevistas, fue difícil que los/as entrevistados/as pudieran identificar y definir las estrategias que implementaban en torno al uso de las tecnologías. Esto nos permitió 
entrever la dificultad para discernir que, muchas de las conductas que ellos implementaban en la cotidianidad, también eran estrategias. En otras palabras, los adultos no reconocen estrategias en la crianza de los niños/as en cuanto al uso de la tecnología. Consideramos que la conducta de los adultos es fundamental para guiar la crianza de los/as infantes, por lo cual, el poder identificar, construir y deconstruir estas estrategias permitirá que la misma se desarrolle más saludablemente. Es decir, las estrategias que los mayores elijan para regular el uso de las tecnologías les enseñarán a los/as infantes, no sólo en qué momentos usarlas, sino que además les brindará información sobre lo que sus adultos de referencia creen apropiado acorde a la edad y para un crecimiento más saludable. Es por esto, que consideramos que un aumento de la creatividad, a la hora de plantear las estrategias de socialización, no sólo generará nuevas alternativas y nuevas propuestas de actividades para los/as infantes, sino que también se acercará a aquello que los adultos consideran como deseable en relación al uso de las pantallas. Así mismo, es notable la poca cantidad de padres, madres, o tutores que eligen como estrategia el promover otras actividades en los/as niños/as o jugar con ellos en sus tiempos libres, así como otros/as que mencionan que al regresar a la casa deben seguir trabajando, por lo que no pueden compartir tiempo con los infantes.

Si entendemos por Uso de Tecnologías a todo contacto cotidiano con diferentes aparatos tecnológicos, podemos afirmar que casi el total de los/as infantes de la muestra (el 90\%) hacían uso de las tecnologías. La edad de iniciación de este uso estuvo situada entre los primeros meses de vida y los 5 años de edad. Resultó significativo que, en la mitad de los/as infantes de la muestra, la exposición a las tecnologías se originó con tan solo unos meses de vida, y que establecieron un contacto diario con dispositivos electrónicos. Según las recomendaciones de la OMS, desde el nacimiento hasta los dos años de edad, no debe existir exposición frente a ningún tipo de pantalla. Para los/as niños/as de 2 años a 4 años, el tiempo indicado, no debe exceder una hora; cuanto menos, mejor (Organización Mundial de la Salud, 2019). De dichos datos, podemos destacar la importancia de brindar información a los adultos sobre las consecuencias del uso de tecnologías a temprana edad y con tanta frecuencia. La información es una herramienta de sumo valor a la hora de tomar decisiones en cuanto a la crianza.

Las principales actividades que realizaban en internet eran prácticas lúdicas, las mismas incluyeron no sólo jugar a videojuegos sino también la búsqueda de vídeos, de imágenes o diferentes contenidos. El juego es un espacio en el que los/as niños/as incorporan reglas y pautas sociales, es decir, también es una instancia de aprendizaje y de socialización (Trinidad y Zlachevsky, 2013). En relación a esto, se puede afirmar que las tecnologías son una herramienta útil para el desarrollo de lo lúdico en la infancia, siempre y cuando el uso sea dentro de los parámetros de salud y de seguridad. En este sentido, es necesario que los adultos asuman un rol más activo, como mediadores del uso que hacen los niños y niñas de las tecnologías.

En lo que respecta a los adultos de la muestra, todos presentaron un alto nivel de manejo de las tecnologías. Se puede pensar que esto les permitiría, a su vez tener un control más estricto del uso que hacen los/as niños y niñas de internet; así como también cooperar con los/as infantes en actividades en torno a las tecnologías, propiciando cambios favorables en las interacciones familiares (Barrera Valencia, y Duque Gómez, 2014). A nuestro entender, puede resultar útil realizar actividades en familia, utilizando las tecnologías como recurso o herramienta para aprender o 
divertirse, y a la vez compartir. Además, teniendo en cuenta que la familia es uno de los principales agentes de socialización en la vida de los/as infantes, entendemos que son los adultos significativos, quienes pueden enseñarles a los/as niños/as a hacer un buen uso de las nuevas tecnologías; ya sea sobre qué contenido ver, en qué momentos, con qué finalidades, en cuanta cantidad, etc.

Por último, cabe mencionar que los modos de interacción y la dinámica familiar, se vieron modificados debido a la implementación del uso de tecnologías. Es indudable que todas las familias presentadas en las entrevistas han tenido algún conflicto en torno al uso de las tecnologías; en todos los casos, se daban entre los adultos y los/as infantes, es decir, en el holón paterno-filial. Sin embargo, también nos parece importante resaltar que estos conflictos, también pueden presentarse dentro del holón conyugal. Por ejemplo, una de las entrevistadas, relató que su marido veía televisión con frecuencia y ella, intentaba restringir constantemente el uso de las tecnologías en su hija. Estas contradicciones en la pareja, les transmiten un doble mensaje a los/as infantes, generando pautas ambiguas y confusas. Es decir, se puede observar que, a pesar de tener conocimientos, la mayoría de las familias, manifestaron tener conflictos en función del uso de las tecnologías, demostrando así, que este saber hacer facilita, pero también tensiona estos vínculos (Duek y Benitez Largui, 2018).

Por otro lado, nos parecía relevante retomar los aportes teóricos en relación a los riesgos y beneficios que traen aparejados el uso de las tecnologías en las infancias, ya que fue una temática que ha aparecido espontáneamente en las entrevistas, aún sin ser una de las variables estudiadas en la presente investigación. En el discurso de los adultos de la muestra, en general, las concepciones respecto a esto, estaban orientadas en relación a preocupaciones sobre los peligrosos contenidos a los que pueden acceder los infantes, así como el contacto con extraños y la posibilidad de que se genere un uso desmedido. Algunas familias también mencionaron que consideran que se pierden los contactos comunicacionales, ya que las horas o momentos para compartir en familia, terminan siendo destinadas, en su gran mayoría, al uso individual de las tecnologías, imposibilitando el diálogo u otro tipo de interacciones familiares. Además, se vislumbró, por parte de los adultos a cargo, la concepción de que el uso de tecnologías en menores, les dificulta la creatividad y la estimulación para el juego, siendo esta una de las explicaciones que los padres, madres o cuidadores eligen para explicarles los motivos de porque no siempre es recomendable usar los aparatos tecnológicos.

En contraposición a lo anteriormente mencionado, con respecto a los beneficios encontramos que los adultos también pudieron dilucidar aspectos positivos de la utilización de las tecnologías, no sólo como un nuevo medio de aprendizaje y entretenimiento, sino también como benefactor de las relaciones familiares, de las habilidades comunicacionales interactivas y simbólicas, y como estimulador del pensamiento creativo y de la respuesta sensorial.

Frente a estas contradicciones, consideramos de real importancia destacar que todo uso llevado a las polaridades del mismo puede devenir en riesgo, por lo cual, es imprescindible la función de los adultos a la hora de enseñar y supervisar el uso que realizan los/as niños/as de las tecnologías, para poder sacar provecho de los beneficios, sin que estos se conviertan en riesgos, debido a un uso inadecuado.

En conclusión, cabe destacar la gran presencia e importancia que cobran las tecnologías en las dinámicas familiares actuales y las transformaciones que estas generan en las mismas. Entendiendo a la dinámica familiar como el entramado de 
relaciones e interacciones que suceden en el seno de la familia y que ésta depende de las negociaciones, los conflictos, la colaboración, el intercambio y el poder de cada uno de los miembros (Gallego Henao, 2012), es posible incluir a las tecnologías como un nuevo integrante en las familias. En base a la apropiación que las familias hacen de las tecnologías, se observa cómo influyen en la manera de relacionarse, sirviendo muchas veces como vehículo de cohesión en esta interacción; en torno a ellas giran las tramas vinculares, se construyen nuevas parentalidades y nuevas configuraciones familiares.

Actualmente estamos atravesando un contexto complejo y diverso que emergió tras la irrupción de una pandemia, y que aún sigue transformándose día a día. La vida cotidiana se ha visto alterada ya que, los espacios, las relaciones y el tiempo se han reinventado. El confinamiento ha puesto en tensión prácticas, acciones y actitudes; ya sea porque han sido mantenidas, modificadas o suspendidas. Esto pone en vista que la realidad es dinámica e impredecible; es singular y plural; lo macro y lo micro interactúan entre sí; se reproducen, pero también se reconfiguran (Guizzo, Marcello y Müller, 2020). En consonancia con esto, la tecnología se ha vuelto el principal medio de socialización, de aprendizaje, de trabajo y de entretenimiento y el hogar, el escenario donde esto sucede. Aguilar Gordón (2020) enuncia que las familias se han convertido en el centro fundamental del proceso educativo, se han vuelto responsables del control y del manejo de actitudes, comportamientos y aprendizajes y les corresponde la identificación de estilos de aprendizaje, el control del tiempoespacio, el seguimiento de las habilidades relacionales, socioemocionales, el acompañamiento y el cultivo de valores. En otras palabras, se ha vuelto tarea casi exclusiva de las familias, estimular y garantizar el bienestar físico, emocional y psicológico de los niños y las niñas.

Teniendo en cuenta que la investigación se realizó en el año 2019 y estuvo enmarcada en una realidad social completamente distinta, consideramos pertinente mencionar la importancia que adquieren los resultados obtenidos en dicho trabajo. Por un lado, porque nos permiten seguir investigando respecto a esta temática y replicar este estudio post pandemia, comparando los resultados. Y, por otro lado, nos invita a repensarnos en relación a las nuevas definiciones tempo-espaciales que se están configurando a partir de la virtualidad; tanto en ámbitos laborales, educacionales, como familiares, siendo estos últimos los que nos competen. Si contemplamos que las tecnologías, de alguna manera, se incorporan como un nuevo integrante en las familias, ¿Qué relevancia cobra esto en un momento en el que los tiempos y los espacios mediados por la virtualidad, son continuos? Sería interesante, pensar en cómo se estructuran y desarrollan las prácticas parentales y familiares, en este nuevo contexto; en el que nos encontramos habitando un mismo espacio con todos los miembros de la familia, lugar también en el que realizamos actividades de ocio, trabajamos, descansamos, estudiamos y nos vinculamos. Frente a esto nos preguntamos ¿Es posible establecer pautas transaccionales y estrategias de socialización respecto al uso de tecnologías, en familias que hoy se encuentran necesariamente e inevitablemente atravesadas por las tecnologías, en todas sus formas? Consideramos que las familias tendrán el desafío de flexibilizarse frente a esta "nueva realidad", buscando construir sus prácticas desde lo que consideren más apropiado para el desarrollo de sus miembros. 
A modo de cierre, retomando el concepto de dinámica familiar, como el entramado de relaciones e interacciones que suceden en el seno de la familia y que depende de las negociaciones, los conflictos, la colaboración, el intercambio y el poder de cada uno de los miembros (Gallego Henao, 2012), es que nos resulta interesante reflexionar acerca de las infinitas formas que pueden adquirir las dinámicas familiares, pudiendo haber, tantas dinámicas como familias. Frente a este nuevo contexto en el que nos encontramos inmersos/as hoy, en el que la tecnología cobra cada vez un lugar más central en nuestras vidas, las dinámicas familiares, inevitablemente y necesariamente, se ven afectadas. Es decir, a medida que la realidad se transforma y la tecnología se incluye como un nuevo integrante de las familias, los vínculos, las funciones, los límites, las jerarquías y las interacciones también irán modificándose.

\section{Referencias}

Aguilar Gordón, F. D. R. (2020). Del aprendizaje en escenarios presenciales al aprendizaje virtual en tiempos de pandemia. Estudios pedagógicos (Valdivia), 46(3), 213-223.

Andolfi, M. (1984). Detrás de la máscara familiar. Buenos Aires: Amorrortu.

Arámbula-Román, Î̃iguez y Solís-Cámara, 2012, en Córdoba, J. (2014). Estilos de crianza vinculados a comportamientos problemáticos de niñas, niños y adolescentes.

Arza, J. (2008). Familia y nuevas tecnologías. Navarra: Consejo Audiovisual de Navarra. Recuperado de http://www. navarra. es/NR/rdonlyres/172CA2032C36-4D8B-AB66-CD9A36956BE4/117076/FamiliayNuevasTecnologias. pdf.

Barrera Valencia, D., \& Duque Gómez, L. N. (2014). Familia e internet: consideraciones sobre una relación dinámica. Revista Virtual Universidad Católica del Norte, 1(41), 30-44.

Córdoba, J. (2014). Estilos de crianza vinculados a comportamientos problemáticos de niñas, niños y adolescentes.

Domínguez Fernández, G., Martínez Jaén, A., \& Ceballos García, M. J. (2017). Educar la virtualidad. Pixel-Bit Revista de Medios y Educación, 50, 187-199.

Duek, C. (Ed.). (2014). Juegos, juguetes y nuevas tecnologías. Capital Intelectual.

Duek, C., \& Larghi, S. B. (2018). Infancias y tecnologías en Argentina: interacciones y vínculos intergeneracionales. Nómadas, (49), 121-135.

Espinal, I., Gimeno, A., \& González, F. (2006). El enfoque sistémico en los estudios sobre la familia. Revista internacional de sistemas, 21-34.

Froufe, S. (1995). El protagonismo de la familia en la transmisión de los valores sociales. Documentación Social. Revista de Estudios Sociales y de Sociología Aplicada, 98, 61- 72.

Gallego Henao, A. M. (2012). Recuperación crítica de los conceptos de familia, dinámica familiar y sus características. Revista Virtual Universidad Católica del Norte, 1(35), 326-345. 
García-Piña, C. A. (2008). Riesgos del uso de internet por niños y adolescentes. Estrategias de seguridad. Acta pediátrica de México, 29(5), 272-278.

Guizzo, B. S., Marcello, F. D. A., \& Müller, F. (2020). La reinvención de la vida cotidiana en tiempos de pandemia. Educação e Pesquisa, 46.

Hernández Sampieri, R., Fernández Collado, C., \& Baptista Lucio, P. (2010). Metodología de la investigación (Vol. 3). México: McGraw-Hill.

Iriarte Díazgranados, F. (2011). Los niños y las familias frente a las Tecnologías de la Información y las Comunicaciones (tics). Psicología desde el Caribe, 208-224.

De Jong, E., Basso, R., Paira, M., \& García, L. E. (2009). Familia: representaciones y significados. Editorial Espacio. Bs As.

Martínez-Piñeiro, E., Couñago, E. V., \& Barujel, A. G. (2018). El papel de la familia en la construcción de la competencia digital. RISTI-Revista Ibérica de Sistemas e Tecnologías de Informação, (28) 1-13.

Minuchin, S. (1982). Familias y terapia familiar. Barcelona: Gedisa.

Minuchín, 1986, Andolfi, 1993; Musitu et al., 1994, Rodrigo y Palacios, 1998 en Espinal, I., Gimeno, A., \& González, F. (2006). El enfoque sistémico en los estudios sobre la familia. Revista internacional de sistemas, 21-34.

Organización Mundial de la Salud. (2019). Para crecer sanos, los niños tienen que pasar menos tiempo sentados y jugar más. Recuperado de: https://www.who.int/es/news-room/detail/24-04-2019-to-grow-up-healthychildren-need-to-sit-less-and-play-more

Prensky, M. (2010). Nativos e inmigrantes digitales. Distribuidora Sek.

Remache Delgado, E. C. (2014). Estilos de crianza en niños y niñas de 0 a 3 años de edad.

Romero, W., Bologna E. (2013). Capítulo 7: Técnicas de muestreo. En E. Bologna, Estadística para Psicología y Educación (tercera edición). Córdoba: Brujas.

Sierra, E. J. (2006). El niño tecnológico: Un perfil educativo. Revista de investigación, (60), 119-131.

Trinidad, R., \& Zlachesvsky, N. (2013). Jugar en contextos tecnológicos: uso y disfrute de internet por niños y niñas de 8 a 10 años en Argentina, Paraguay y Perú. Contratexto, (021), 211-227.

Vodopivec, J. L., \& Samec, P. (2013). Uso de tecnologías en el entorno familiar en niños de cuatro años de Eslovenia. Comunicar: Revista científica iberoamericana de comunicación y educación, (40), 119-126.

Yubero, S., Larrañaga, E., Navarro, R., \& Elche, M. (2018). Padres, hijos e internet. Socialización familiar de la red. Universitas Psychologica, 17, 88-100. 